\title{
Sylow 2-子群为交换的有限单群的刻划
}

对 Sylow 2-子群为交换的有限单群, J. H. Walter 证明了如下有名的定理.

引理 1 若 $F$ 是 Sylow 2-子群为交 换的有限非 abel 单群, 则下述结论之一成 立:

(1) $F \cong P S L(2, q), q>3, q \equiv 3$, $5(\bmod 8)$ 或 $q=2^{n}, n \geqslant 2$;

(2) $F \cong J_{1}$;

(3) $F \cong R(q), q=3^{2 m+1}, m \geqslant 1$.

设 $G$ 是有限群, $\pi_{e}(G)$ 为 $G$ 中所有元的 阶之集. 在文献 [1]中作者证明了 $G \cong F(F$ 为上述引理中情形 (1)或 (2)) 当且仅当

$$
\pi_{e}(G)=\pi_{e}(F) \text {. }
$$

本文中我们证明了 $G \cong R(q), q=3^{2 m+1}$, $m \geqslant 1$, 当且仅当 $\pi_{\theta}(G)=\pi_{c}(R(q))$. 于 是有如下定理:
定理 设 $G$ 是有限群, $F$ 为 Sylow 2子群为交换的有限非 abel 单群. 则 $G \cong F$ 当且仅当 $\pi_{e}(G)=\pi_{c}(F)$.

下述引理在本文的证明中起 着 关 键 作 用.

引理 2 设 $N$ 为有限群 $K$ 的正规 3-子 群, $H=K / N=[M] Q$ 为 Frobenius 群, 且核 $M$ 为可换, $Q \cong \mathrm{Z}_{6}$, 再设 $M$ 无不动点地 作用在 $N$ 上,则 $N=1$ 或 $18 \epsilon \pi_{e}(K)$.

\section{参 考 文 献}

[1] 施武杰,数学进展, 1987, 16(4): 397-401.

R. Brandl

(Mathematisches Insititut, Würzburg, Germany) 施武杰

(西南师范大学数学系, 重庆 630715)

\section{纳米小孔和纳米 $\mathrm{X}$ 光显微术}

继扫描隧道显微镜 (STM) 原子力显微 镜 (AFM) 之后; 光学显微术也采用纳米探 头 (probe) 扫探而获得 $\sim 12 \mathrm{~nm}^{[1]}$ 以至 $2 \mathrm{~nm}^{[2]}$ 分解的结果, STM 和 AFM 采用的探头是 钨针, 光学显微术采用的则是光纤针, 用腐蚀 可获得极细的尖端 (达 $1 \mathrm{~nm}$ 量级). 这种方 式不能用于 $x$ 光.

迄今对 X 光波段曾建议过多种显微术但 没有一种能达到纳米级分辩 ${ }^{[3]}$. 这是由于难 以制成高精度的光学系统以成象. 而过去的 $\mathrm{X}$ 光全息都是用 Gabor 式全息记录,从而要 求记录介质具高分辩能力 ${ }^{[4]}$. 由于生物样品 在水窗 $(2-4 \mathrm{~nm})$ 有高反差, $\mathrm{X}$ 光显微术若 比汰到高分辨将是十分有意义的.
因此我们建议用纳米针孔作为探头, 使 $\mathrm{X}$ 光扫描地透过样品, 形成纳米分辨 $\mathrm{X}$ 光显 微术. 由于针孔直径接近波长, 也仍然须贴 近样品扫描 (Near field scanning). 制作 纳米针孔看来有多种可能性, 例如在金属薄 脱上用纳米铇针尖作电火花加工，再如将金 属薄膜上用激光加工成 $1 \mu \mathrm{m}$ 小孔(这是成熟 技术)，然后用电镀或真空镀膜加厚填满 小 孔(腐蚀的逆过程).

作者曾提出非觉余全息记录方案的此时 记录介质的分辨能力与分辨物体细节的能力 无关, 但此时要求有一个点参考光束源, 采用 纳米针孔作为参考点（它可以用 Fresnel 诐 带片聚焦 X光束作照明), 就可以用非午余 X 
光全息照相术记录物体细节达到纳米 分'游, 而且得到的是立体结构, 优于纳米探头扫描 成象技术. 作者并分析了非罙余记录对光源 相干度的要求, 表明采用同步辐射 $\mathbf{X}$ 光源完 全可以满足 $\mathrm{X}$ 光非午余全息记录的要求.

\section{参文嗝}

[1] Betzig, E. et al., Science, 1991,251:1468;1992,
257: 189.

[2] Lases Focus World, 1993, 29(4): 44; Ohtsu, M., Photon STM, Invited Lecture of OITDA 2nd. International Exchange Forum, 1992.

[3] Attwood, D., Phys. Today, 1992, 45 (8): 24.

[4] McNulty, I. et al., Science, 1992, 256: 1009; Rev. Sci. Instrum., 1992, 63: 557.

[5] 王之江, 科学通报, 1979, 24(8): 719; Wang Zhijiang, OSA Annual Meeting, 1992, MG4. 王之江

(中国科学院上海光学精密机械研究所, 上海 201800)

\section{状 态树的 优化生 成}

人类的任何一项有意义的活动都是 “有 的放矢”的,务期达到既定的目标. 然而, 对 某些复杂的问题而言, 目标状态并非显而易 见. 由于计算机的存储容量有限, 更由于提 高问题求解效率的需要, 人们不宜罗列出待 求解问题的全部状态, 而应设法迅速得出其 目标状态集合(目标集). 为此, 作者提出了 状态树的优化生成法.

大凡复杂的任务 $R$ 都是由多项子任务 $r_{i}$ $(i=1,2, \cdots, n)$ 组成的, 而子任务间的相 互关系不外乎三种:

（1）相关型（包括同时发生和顺序发生 两个子类);

(2) 互斥型;

(3) 独立型.

当目标状态集合是相容的子 任务状 态 时,我们可以用勭枝法优化生成状态树.

则 $1 R$ 由子任务 $r_{1}, r_{2}, r_{3}$ 组成, 子任

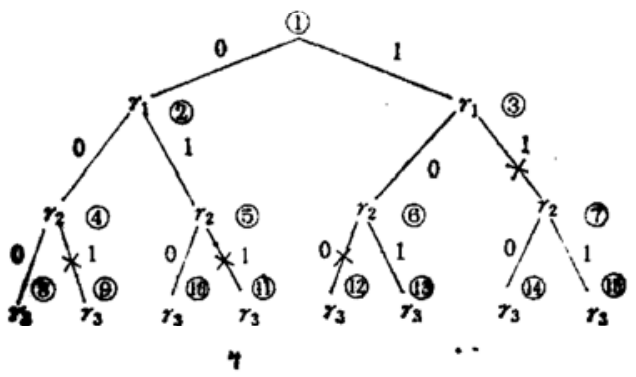

图 1 状态树的剪枝生成
务间的约束条件为 $r_{1} \Rightarrow \bar{r}_{2}, r_{1} \Leftrightarrow r_{3}$. 即 $r_{1}$ 同 $r_{2}$ 互斥, $r_{1}$ 同 $r_{3}$ 相关. 于是, 其状态树 如图 1 所示.

在图 1 中, 枝的赋值为 0 表示即将到达 的那个节点所代表的子任务将不出现, 而枝 的䟼值为 1 的含义恰好相反，表示相应的子 任务会出现. 从根节点到叶节点的每一条路 径相应于子任务的一种组合, 即本问题的一 个状态. 对于本问题而言, 共有 $2^{3}=8$ 个状 态, 分别相应于从根节点到 8 个叶节点的 8 条路径. 在本题给定的约束条件下, 或者说 在所给出的启发信息的引导下, 通过如图中 $\times$ 号所示的剪枝,我们得到的目标集为

$$
G=\left\{r_{1} \bar{r}_{2} r_{3}, \bar{r}_{1} r_{2} \bar{r}_{3}, \overline{r_{1} r_{2} r_{3}}\right\},
$$

即图中的三条路径(1) $\rightarrow$ (3) $\rightarrow$ (6) $\rightarrow$ (13)、(1) $\rightarrow$ (2) $\rightarrow$ (5) $\rightarrow$ (10)和 (1) $\rightarrow$ (2) $\rightarrow$ (4) $\rightarrow$ (8)所代表的三种状 态构成了本问题的目标集.

在完备的约束条件下（即充分的启发信 息指引下),勖枝法生成的效率为 $100 \%$ 。例 1 便属于这类情况. 我们没有遍历 8 条可能 的路径便找到了所有的目标状态. 即便是有 关的约束不完备, 利用这里的蒥枝法也可显 著地提高生成目标集的效率.

\section{涂承宇 涂我媛}

(北京工业大学电子工程学系, 北京 100022) 